East African Medical Journal Vol. 86 (Supplement) December 2009

APPROACHES TO ADVANCING BLOOD SAFETY THROUGH HAEMOVIGILANCE: A REVIEW

J. W. Mwangi, MBChB, MMed (Path), MSc (HSM), D. Kimani, MBChB, MPH, Center for Disease Control and Prevention, Kenya, P. O. Box 42419-00100, Nairobi, Kenya and M. Oduor, MBChB, MMed (Path), National Blood Transfusion Services, Ministry of Medical Services, P. O. Box 29804-00202, Nairobi, Kenya

Request for reprints to: Dr. J. W. Mwangi, P. O. Box 42419-00100, Nairobi, Kenya

\title{
APPROACHES TO ADVANCING BLOOD SAFETY THROUGH HAEMOVIGILANCE: A REVIEW
}

\author{
J. W. MWANGI, D. KIMANI and M. ODUOR
}

\begin{abstract}
Background: Blood transfusion is always associated with some level of risk. Haemovigilance is a risk monitoring system integral to the practice of transfusion medicine whose ultimate purpose is to improve the quality and safety of transfusion therapy.

Objective: To examine the contribution of haemovigilance to blood safety, including the approaches that some countries have taken to institute haemovigilance, and explore routes through which countries without such systems can achieve them.

Data sources: The internet and journals on the topic of haemovigilance and development of haemovigilance systems in the English language.

Data selection: Reputable journals on the topic of haemovigilance were examined for abstracts and papers. Abstracts based on known credible and distinguished sources were selected.

Data extraction: Information on haemovigilance and the processes of developing haemovigilance in various countries was reviewed.

Data synthesis: The information from selected papers and abstracts was used for writing this paper.

Conclusion: Varying processes for haemovigilance have been adopted by different countries. The more advanced systems have national/regional coordinating mechanisms. Availability of haemovigilance data has given transfusion services a clear understanding of problems associated with transfusion that need to be solved so as to improve transfusion safety. Although countries in sub-Saharan Africa have made considerable progress in enhancing blood safety in the recent past, nationally coordinated haemovigilance systems are lacking. Focus on haemovigilance systems is considered the next frontier to be conquered in enhancing blood safety in the region.
\end{abstract}

\section{INTRODUCTION}

The primary objective of national blood transfusion services (BTS) is to ensure safety, adequacy, accessibility and efficiency of blood supply through establishment of efficient, cost-effective and coordinated national systems. BTS include the chain of activities beginning from the mobilisation and selection of blood donors through blood processing to the transfusion of patients. The benefits of blood transfusion are indisputable with blood now being widely regarded as a drug that contributes to the successful management of many patients. The transfusion of blood, blood components and products is however not risk free. The threat of infectious agents entering the blood supply is not static and may evolve as new pathogens emerge or as old ones change their epidemiological pattern (1). Indeed blood is well known to be an efficient vehicle for transmission of amongst other infections hepatitis, HIV and malaria. Other than the infectious risks, transfusion may be associated with complications related to exposure to foreign antigens, altered chemical/temperature balance and anaphylaxis. The term "safe blood transfusion" implies an outcome that is totally beneficent and nonmaleficent to the patient. Safe blood practice is dependent on several factors including selection of volunteer blood donors with low risk for transfusion transmissible infections (TTI), aseptic collection of blood donations, use of sensitive screening tests for TTI, appropriate cold chain maintenance, accurate cross-matching, and elimination of unnecessary transfusions through appropriate and rational clinical use of blood and blood products. The process of monitoring for the lapses at any one of 
these stages is termed haemovigilance. To promote blood safety, the World Health Organisation (WHO) encourages member countries to establish nationally coordinated haemovigilance programmes focused on improvement of transfusion practices (2).

Types of haemovigilance systems: Haemovigilance is an important risk monitoring system integral to the practice of transfusion medicine whose ultimate purpose is to enhance the quality and safety of transfusion therapy through continuous improvement of transfusion standards. All processes throughout the chain of events from donor selection and phlebotomy to the post-transfusion phase are monitored for the occurrence of adverse or near-miss events. Both transfusion recipients and blood donors should be followed up in this process so as to detect any immediate and long-term effects of transfusion and blood donation respectively. The conventional system for haemovigilance is event notification. Reporting of adverse, unexpected or near miss events or reactions in blood donors or recipients enables identification of gaps in quality systems management and consequently prompts the search for corrective measures required to ensure safe blood practice.

A less common form of haemovigilance is the direct observation system, based on direct observation of transfusions by appropriately trained technical staff at the patients' bedside and verification of adherence to standard operating procedures $(3,4)$.

"Look back" is yet another form of haemovigilance. It involves efforts to identify recipients of products from a particular donor usually after the donor is found to have a condition that may have put the recipients at risk of a TTI such as HIV or hepatitis. The success of look back depends on well established traceability systems enabling the identification of the recipient of any given blood component and conversely the tracing all blood components received by a patient. Whereas these three approaches focus on clinical outcomes of blood donation or transfusion, a manufacturing/industry quality process approach emphasizes compliance to standard operating procedures throughout the transfusion chain.

Analysis of haemovigilance systems: Haemovigilance systems have been established in several countries with significant benefits to the BTS. Transfusing facilities in the USA are obliged to inform the Food and Drug Administration (FDA) of transfusionrelated deaths. Hospital accrediting bodies such as The Joint Commission on Accreditation of Healthcare Organizations (JCAHO) and the College of American Pathologists (CAP) additionally require reporting or at a minimum documentation of all adverse transfusion events. The Centers for Disease Control and Prevention's National Healthcare Safety Network (NHSN) recently instituted a voluntary internet based haemovigilance module within its broader biovigilance programme to track and coordinate national reports of adverse events in transfusion recipients and blood donors (5). The main cause of reported deathsis transfusion-related acutelunginjury (TRALI) (30\%), followed by haemolytic transfusion reactions $(16 \%)$ and bacterial contamination (16\%) (6). Retrospective analyses carried out separately by Linden et al (6), Honig and Bove (7) and Sazama (8) and on transfusion incidents occurring in the United States in the 1970s-1980s concordantly found that the incidence of fatal acute haemolytic reactions was one case in every 600,000 units transfused. An analysis of transfusion errors in New York State in a 22-month period in 1990-1991 showed that $43 \%$ (45/92) of the errors, of which $58 \%$ occurred outside the transfusion structure, were due to incorrect identification of the patient and/ or the unit of blood (6).

Two main types of risk monitoring systems exist in Europe; the voluntary (United Kingdom, Denmark, Greece, Ireland, Italy) and the obligatory (France, Germany, Switzerland). In the United Kingdom (UK), reports are monitored through a centralised voluntary, anonymous and independent process referred to as Serious Hazards of Transfusion(SHOT) $(9,10)$. SHOT aims to educate blood transfusion practitioners through annual reports and to provide an evidence base to support decisions, guidelines, clinical training and improvements in transfusion practices. Analysis of data collected by SHOT on the occurrence of Transfusion Related Acute Lung Injury (TRALI) linked its use of plasma from multiparous females leading to discontinuation of use of plasma from these donors (11).

France has a national haemovigilance system with two separate but parallel institutions. The French Health Products Safety Agency-Agence Francaise de SécuritéSanitaire des Produits deSanté-(AFSS-APS) has a regulatory function while executive functions are vested with Etablissement Francais duSang(EFS) (12). There is a legal obligation to notify all adverse events, irrespective of the severity and clinical consequences of the error. Near-miss events however, do not need to be reported in the UK. In the years 1994-1998, the French haemovigilance system recorded a mortality rate equivalent to that found by Linden et al (6) in New York State, with one death for every $1,802,739$ red cell products transfused.

The Danish Registration of Transfusion Risks (DART) is a part of the Danish haemovigilance system that covers registration of collected, produced and transfused blood components, and complications in connection with transfusion of these. In the first five years of data collection in the Danish haemovigilance system, half of the severe events $(53 \%)$ followed the 
transfusion of an incorrect blood component and the other half were due to immunological complications $(43 \%)$. Only $4 \%$ of the reported events concerned transfusion transmitted infections (TTI) (13). In Germany, every adverse transfusion reaction must be reported by the transfusion centres to the PaulEhrlich-Institute(PEI). Transfusion errors occasioned by inappropriate use of blood components and not by the properties of the product itself are however not reported to the PEI $(9,10)$.

Retrospective analysis of data collected between 1999 and 2003 at a hospital in Switzerland found that the incidence of transfusion reactions was $4 \cdot 19$ per 1000 blood products (14). Whereas some countries report only serious events, others like France, report all events including "near-miss" events. A serious adverse event is defined as an unintended occurrence associated with the collection, testing processing, storage and distribution of blood orblood components that might lead to death or life threatening, disabling or incapacitating conditions for patients or which results in, or prolongs, hospitalisation or morbidity. Near-miss events refer to events that did not result in injury but had the potential to do so other than for recovery stepsallowing for interruption and correction of the error(s) and dysfunction(s). Data collected in countries with obligatory reporting systems reveals that the proportion of near-miss events, with respect to the total number of adverse transfusion events, is $90 \%$ (3). Near-miss events are an important indicator of risk gaps in the transfusion system and present opportunities for improvement without any associated potential punitive measures (15). Voluntary reporting systems generate significantly fewer reports than obligatory systems. For example in $2001,8.5$ notifications $/ 100,000$ blood components distributed in the UK were made in contrast to 325.2/ 100,000 blood components distributed in France (3). This finding indicates that voluntary reporting denies the haemovigilance system information important to the enhancement of national blood safety. Legislation in the European Union (Directive 2002 EC) requires adverse event reporting stating that "Member States shall ensure that any serious adverse events (accidents and errors) related to the collecting, testing, processing, storage and distribution of blood and blood components which may have an influence on their quality and safety, as well as any serious adverse reactions observed during or after transfusion which may be attributed to the quality and the safety of blood and blood components are notified to the competent authority" (16).

Although based on different ways of collecting data, the two major European haemovigilance systems do contain important information useful for comparison of the data collected, itsinterpretation and evaluation of the corrective actions taken. In contrast to the USA in which only fatal errors are notified, the
European systems collect data on both fatal and nonfatal transfusion errors. Haemovigilance systems in South Africa and New Zealand are modeled on the UK SHOT system. Although it has been estimated that $5-10 \%$ of HIV transmission in Africa is by contaminated blood transfusions, according to the WHO Global data base on blood safety, in 2004 the rate of full implementation of blood policies and quality management systems in the WHO-AFRO region was very low at $17 \%$ and $20 \%$ respectively $(17,18)$. Further, a recent review of blood safety in sub-Saharan Africa reported that many countries in Africa donothave an effective haemovigilance system (19). These findings are corroborated by a hospital based study in Cameroon which reported that half of transfusions within the hospital were associated with an unfavorable outcome, predominantly febrile reactions and urticaria but also acute intravascular haemolysis, circulatory overload, post-transfusion HIV infection and death (20). Additionally, a study in Kenya in 1994 estimated that $2.0 \%$ of transfusions transmitted HIV (21).

Proposal for initiating haemovigilance: Haemovigilance can be instituted through both top-down and bottomup approaches. In the latter approach, individual health facilities should institute transfusion safety monitoring systems and link to a centrally coordinated national or regional haemovigilance system that provides feedback to BTS and health ministries. Whereas the blood banking and processing aspects can be easily monitored through a technical audit process, donor care and appropriate blood use are more effectively monitored through donor satisfaction surveys and peer reviews on appropriate blood use. This calls for a model that combines evaluation of both quality processes and clinical outcomes. Peer review is best achieved through hospital transfusion committees (HTC). The HTC has a primary responsibility to monitor and evaluate the clinical use of blood within the health facility so as to ensure patient safety and judicious use of the blood supply. Guidelines for the rational use of blood form the basis of the monitoring function of the HTC which should also organise clinician awareness training programmes to promote strategies for rational use of blood. HTCs monitor transfusion orders, quantities transfused, indications for transfusion, occurrence of adverse events and their investigation. At facility level, selection of events to be monitored may include haemolytic transfusion reactions, transfusion related lung injury (TRALI), bacterial contamination, anaphylactic reactions, post transfusion purpura, well recognised TTIs, emerging TTIs, near miss events, minor and long-term morbidity and deaths associated with transfusion as well as processes such as blood donation, laboratory testing and 
blood processing, ordering, bedside transfusion and blood utilization. Investigations of such reactions and consequent corrective measures should span the BTS chain incorporating both the health facility and the National Blood Transfusion Services. In addition to the data on reactions to transfusion, errors of administration, overuse or unnecessary administration of blood and underuse or failure to give necessary transfusion should also be monitored. HTCs serve to bridge the gap between blood banks and transfusing facilities ensuring that safe and efficacious transfusions are given. It has been shown that the implementation of transfusion audits can significantly reduce the inappropriate use of blood products (22).

The second step in this approach to haemovigilance is the creation of a national process to receive and harmonize facility based haemovigilance reports, streamline regulations and institute changes beneficial to transfusion practice. Decisions on what aspects of haemovigilance are to be monitored at a national level may be guided by prevailing TTIs, available technology and monitoring capacity. The probable, possible or unlikely attribution of transfusion to these events should be ascertained or excluded through a root cause analysis by a panel of objective reviewers. Many post transfusion deaths are not due to the transfusion but rather consequent to the primary illness. For example, Sazama (8) reported that of 355 fatalities associated with transfusion, 99 wereexcluded from further review because they were unrelated to the transfusion process.

Thorough investigation of adverse events should determine where in the transfusion chain incidents occur, (blood bank, ward, theatre, ICU or casualty), when incidents occur, what causes incidents, which products are most vulnerable and trends in occurrence of adverse events. This reporting system may be voluntary, as in the United Kingdom or enforced by law as in France. Haemovigilance systems that operate with legal backing tend to achieve higher reporting rates (3). Evaluations of voluntary and obligatory systems suggest that regulatory structures are essential for successful implementation of haemovigilance and result in more comprehensive reporting. Evidence from several countries indicates that where outcomes of haemovigilance result in punitive action reporting rates are lower and hence systems that focus on education and process improvement are preferable (22).

As a final step national haemovigilance systems should be linked to an international network to promote global blood product safety and quality. Processes for timely collection of information, disseminating findings and ensuring confidentiality promotesuccess. Countriesinsub-SaharanAfricahave made significant positive achievements in enhancing BTS over the last five years by implementing key
WHO pillars for blood safety such as formulation of policies for BTS stipulating collection of blood only from voluntary donors, quality blood testing and reduction of unnecessary transfusion (23).

\section{CONCLUSION}

Haemovigilance is a measure of compliance to and the effectiveness of quality management systems and an excellent quality indicator for the blood transfusion service. Without haemovigilance, it is impossible to definitively weigh the risk associated with a transfusion and consequently difficult for clinicians to assess the benefit/ risk ratio and counsel pre-transfusion patients accordingly. Haemovigilance has enabled several countries to institute relatively simple measures to improveblood safety with success such as use of wrist bands for patient identification and exclusion of multiparous females from plasma and platelet donations. Despite some progress implementation of WHO recommendations for blood safety, very few countries in Africa have formulated legislation to regulate practice and monitoring of blood transfusion. The time is now ripe for these countries to focus more attention on monitoring and evaluation of BTS including institution of haemovigilance systems. To achieve this data collection, data validation and reporting around blood safety must be strengthened. Evaluation of data from haemovigilance systems should form the basis for development of standards and guidelines for transfusion practice to promote blood safety and limit inappropriate blood use. Availability of haemovigilance data provides a precise delineation of problems associated with transfusion that need to be solved so as to improve transfusion safety. Key players in successful institution of national haemovigilance systems includehealth facility / blood bank based professionals, a national coordinating body, regulatory / policy formulating body.

\section{REFERENCES}

1. Bihl, F., Castelli, D., Marincola, F., et al. Transfusiontransmitted infections. J. Transl. Med. 2007; 5:25.

2. World Health Organization. Aide-memoire for national blood programmes. Geneva, Switzerland 2002.

3. Prinoth, O. Systems for monitoring transfusion risk. Blood Transfusion. 2008; 6: 86-92.

4. Whitsett, C.F. and Robichaux, M.G. Assessment of blood administration procedures: problemsidentified by direct observation and administrative incident reporting. Transfusion. 2001; 41: 581-586.

5. Williams, L. TheUSBiovigilance Network: Improving patient safety and donor health. AABB News. 2009; 11(10): 8-10.

6. Linden, J.V., Wagner, K., Voytovich, A.E., et al. Transfusion errors in New York State: an analysis of 10 years' experience. Transfusion. 2000; 40: 1207-1213. 
7. Honig, C.L. and Bove, J.R. Transfusion-associated fatalities: review of Bureau of Biologics Reports 19761978. Transfusion.1980; 20: 653-661.

8. Sazama, K. Report of 355 transfusion-associated deaths: 1976 through 1985. Transfusion. 1990; 30: 583-590.

9. Williamson, L.M., Cohen, H., Love, E.M., et al. The Serious Hazards of Transfusion (SHOT) initiative: the UK approach to haemovigilance. Vox Sang. 2000; 78 (Suppl 2): 291-295.

10. International Forum. Haemovigilance. Vox Sang. 2006; 90: 207-241.

11. Staropoli,A.TheUSBiovigilance Network: Improving PatientSafety and Donor Health, A. AABB News. 2009; 11: 18-22.

12. Debeir, J., Noel, L., Aullen, J.P., et al. The French Haemovigilance System. Vox Sanguinis. 1999; 77:81.

13. Jorgensen, J. and E. Taaning. DART-A Voluntary System of Haemovigilance in Denmark. Trans. Alternatives Trans. Med. 2003; 5: 260-262.

14. Siegenthaler, M.A., Schneider, P., Vu, D. and Tissot, J. Haemovigilance in a general university hospital: need for a more comprehensive classification and a codification of transfusion-related events. Vox Sanguinis. 2005; 8: 22-30.

15. Kaplan, H.S. Getting the right blood to the right patient: the contribution of near-miss event reporting and barrier analysis. Trans. Clin. Biol. 2005; 12: 380-384.

16. Official Journal of the European Union 8.2.2003, L 33/30. Directive 2002 // EC of the European Parliament and of the Council of setting standards of quality and safety for the collection, testing, processing, storage and distribution of human blood and blood components and amending directive $2001 / 83 /$ EC

17. Tapko, J.B., Sam, O. and Diarra-Nama, A.J. Status of blood safety in the WHO African region: report of the 2004 survey. 2007.

18. Lackritz, E.M. Prevention of HIV transmission by blood transfusion in the developing world: achievements and continuing challenges. AIDS.1998; 12: S81-S86.

19. Tagny, C., Mbanya, D., Tapko, J. and Lefrère, J. Blood safety in sub-Saharan Africa: a multi-factorial problem. Transfusion. 2008; 48: 1256-1261.

20. Mbanya, D., Binam, F. and Kaptue, L. Transfusion outcome in a resource-limited setting of Cameroon: A five-year evaluation International. J. Infect. Dis. 2001; 5:70-73.

21. Moore, A., Herrera, G., Nyamongo, J., et al. Estimated risk of HIV transmission by blood transfusion in Kenya. Lancet. 2001; 358:657-660.

22. AuBuchon, J. The Role of Haemovigilance in Transfusion Safety: Practical Transfusion Medicine (Third Edition), 181-189; YR: 2009; Blackwell Publishing Ltd

23. Centers for Disease Control and Prevention, Progress Toward Strengthening Blood Transfusion Services -14 Countries, 2003-2007. MMWR 2008; 57: $1273-1277$. 